\title{
The incompleteness of physics
}

What is the greatest mathematical achievement of all time? Judged in terms of the consequences for science and engineering, one might argue for the invention of calculus by Newton and Leibniz. Or perhaps the earlier invention of algebra, by unknown Babylonian thinkers, deserves a higher place. If by 'great' we mean 'heroic', then Andrew Wiles' proof of Fermat's Theorem deserves mention. But for sheer intellectual surprise, and alarming insight, it is hard to think of any result that would approach the famous incompleteness theorem of Kurt Gödel.

In 1900, when David Hilbert launched a programme to put all of mathematics on a sound axiomatic footing, few doubted that this would be possible. Hilbert sought to build mathematics up as a logically flawless structure that would be complete, in that every true statement $S$ could be derived from a finite set of axioms, and also consistent, in that it would never be possible also to derive the negation of $S$.

In 1931, however, Gödel demolished this vision of logical tidiness. He proved that Hilbert's dream was impossible, because any system of axioms strong enough to produce arithmetic would necessarily have to be either incomplete or inconsistent. It would always be the case either that some statement $S$ would be at once both provably true and false, or that some statements, although true, would never be derivable from the axioms. Any usefully rich axiomatic system, he showed, is either selfcontradictory or has holes in it.

It is natural to speculate - and many have - as to what Gödel's proof implies for physics. If physics is based on mathematics, and mathematics cannot be ordered consistently in axiomatic terms, is this then true of physics as well? An ancient dream holds that we'll one day discover the simple, foundational laws from which all the possibilities of the physical world logically follow. But maybe we won't. Perhaps, by analogy, no set of fundamental postulates could ever produce a world as rich as ours?

This is apparently the conclusion that Freeman Dyson drew from Gödel's result, and it pleased him. "Gödel proved that the world of mathematics is inexhaustible," he wrote, "and I hope that an analogous situation exists in the physical world... no matter how far we go in the future there will

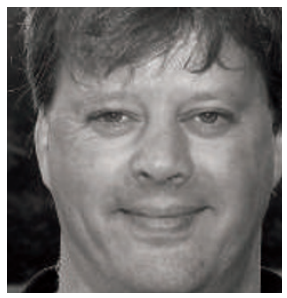

The loss

of logical

foundations might actually be liberating. always be new things... new worlds to explore."

John Barrow now suggests that the logic of the situation may be more delicate than many have supposed (http://arxiv.org/abs/ physics/0612253). It could be, he argues, that not all of mathematics has a counterpart in the physical realm - that the physical world may only make use of the decidable (logically derivable) part of mathematics issuing from some fundamental axioms.

Alternatively, it's possible that the conditions assumed in Gödel's proof don't hold in the physical analogy. For example, the set of fundamental laws of physics might not be finite. "It is always possible," as Barrow puts it, "that we are just scratching the surface of a bottomless tower of laws."

As he points out, Gödel himself suggested another, weirder possibility - that the loss of logical foundations might actually be liberating. It may imply, he suggested, that mathematical intuition will one day be restored as a reliable means for finding truth, and put on a par with the physical senses.

Mark Buchanan

\section{Memories of a friend}

Richard Feynman was my personal friend. Having Feynman for a friend was never relaxing, because he was always 'on'. But it could be, and often was, great fun.

My first experience of Richard came on my first visit to Caltech, for a job interview/seminar in 1965 when I was tying up loose ends in my thesis as a postdoc at the University of Washington in Seattle. Feynman, who went on to win the Nobel Prize in Physics that year, was then unknown to the general public, but he was already a legend among physicists. I hoped that I might catch a glimpse of the great man, even if at a distance.

But when my contact at Caltech, Jim Mercereau, met me at the Los Angeles airport, he asked me if I would mind having lunch with Feynman before going to the campus. I agreed, of course. Lunch was at Feynman's favourite hangout at the time - a topless restaurant in Altadena, just north of Pasadena. We met there and had our lunch. All I can remember from that hour of intense culture shock is thinking to myself, over and over again, "Nobody in Seattle is going to believe this."

That afternoon I gave my seminar, with Feynman in attendance. He peppered me with tough questions, but I must have done all right, because I was hired, and I have spent my entire career since then as a Professor at Caltech. That in turn enabled me

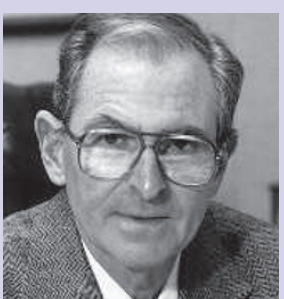

"Nobody in

Seattle is going to believe this." to spend much more time with Richard Feynman.

In the last ten years of his life, Dick underwent a series of operations for stomach cancer, and I became increasingly fearful of losing him. During this time, he was invited to address a group of high-school physics teachers. He turned down the invitation regretfully but when the morning came he was feeling better, and he called me to ask if I would take him there. I did and he was magnificent - his old self, mugging and performing for his audience.

I believe that was the last public appearance Feynman ever made.

David Goodstein 